\title{
Therapeutic Effects and Adverse Drug Reactions are Affected by Icotinib Exposure and CYP2C19 and EGFR Genotypes in Chinese Non-Small Cell Lung Cancer Patients
}

\author{
Jia Chen ${ }^{1}$, Xin Zheng ${ }^{1}$, Dong-Yang Liu ${ }^{1}$, Qian Zhao ${ }^{1}$, Yi-Wen Wu ${ }^{1}$, Fen-Lai Tan ${ }^{2}$, \\ Yin-Xiang Wang ${ }^{2}$, Ji Jiang ${ }^{1}$, Pei Hu${ }^{1 *}$
}

\begin{abstract}
Background: The aim of this study was to evaluate how CYP2C19 affects icotinib and metabolite' exposure, and to determine whether the exposure and EGFR genotype influences survival time, tumor metastasis and adverse drug reactions. Materials and Methods: 274 NSCLC patients who accepted 125mg icotinib/t.i.d. were chosen from a phase III study. Blood samples were obtained in $672^{\text {nd }}\left(4^{\text {th }}\right.$ week $)$ and $1,680^{\text {th }}$ hours $\left(10^{\text {th }}\right.$ week $)$, and plasma was used to quantify the concentration of icotinib and blood cells were sampled to check the genotypes. Clinical data were also collected at the same time, including EGFR genotypes. Plasma concentrations were assessed by HPLC-MS/MS and genotype by sequencing. All data were analyzed through SPSS 17.0 and SAS 9.2. Results: CYP 2C19 genotypes affected bio-transformation from icotinib to M24 and M26, especially in poor-metabolisers. Higher icotinib concentrations $(>1000 \mathrm{ng} / \mathrm{mL})$ not only increased patient PFS and OS but also reduced tumor metastasis. Patients with mutant EGFR experienced a higher median PFS and OS (234 and 627 days), especially those with the 19 del genotype demonstrating higher PR ratio. Patients who suffered grade II skin toxicity had a higher icotinib exposure than those with grade I skin toxicity or no adverse effects. Liver toxic reactions might occur in patients with greater M20 and M23 plasma concentrations. Conclusions: CYP2C19 polymorphisms significantly affect icotinib, M24 and M26 exposure. Patients with mutant EGFR genotype and higher icotinib concentration might have increased PFS and OS and lower tumor metastasis. Liver ADR events and serious skin effects might be respectively induced by greater M20, M23 and icotinib concentrations.
\end{abstract}

Keywords: Icotinib - metabolites - CYP2C19 - EGFR - PFS and OS - liver ADR

Asian Pac J Cancer Prev, 15 (17), 7195-7200

\section{Introduction}

Icotinib is an oral EGFR-TKI agent which is the first home-grown anticancer drug developed by Zhejiang Beta Pharma Inc. (Zhejiang, China) (Tan et al., 2012; Camidge, 2013). The preclinical animal experiments showed that the compound had an anticancer activity in vitro and in vivo whose mechanism is that icotinib can inhibit EGFR activity specifically and competitively through binding to the tyrosine of the EGFR. So inhibiting the activity would block the related signal conduction and thereby significantly inhibit the tumor cell growth (Gao et al., 2013; Mu et al., 2013). Owing to its pharmacological effect, icotinib was approved by CFDA to treat non-small cell lung cancer, and has been market in 2011. Recently, a paper published by Shi et al. (2013) demonstrated that patients taking icotinib was with a longer progressive free survival time (PFS) than those taking gefitinib in a phase III study.

Up to date, icotinib has completed phase I, II and III trials (Wang et al., 2011, Zhao et al., 2011; Shi et al., 2013; Shao et al., 2014). Phase I clinical trial data displayed that excellent tolerance, whose highest safe dose of $1025 \mathrm{mg}$, among healthy Chinese subjects (Liu et al., 2014). Pharmacokinetic study in phase I trial exhibited nonlinear character with saturated absorption and first-order elimination (Wang et al., 2011). From all papers about icotinib, CYP 2C19 genetic polymorphism is the only reported factor produced the metabolism variability, which illustrated AUC and Cmax of icotinib in subjects were 1.56 and 1.41 higher than those in subjects who carrying CYP 2C19*2/*3, and CL/F was 1.55-fold higher (Ruan et al., 2012). But the influence of CYP2C19 polymorphism in NSCLC patients was not further assessed, and some questions still keep puzzling: Whether changing of icotinib exposure would make influences on PD and therapeutic effect and have any relationship with toxicity are still confused. In this study, we tried to analysis the relationship between exposure and therapeutic effect, toxicity in some subjects from a phase III study. 


\section{Materials and Methods}

\section{Patients and blood samples}

274 patients coming from a randomized, doubleblind, head-to-head phase III study (ICOGEN), provided written informed consent before participation in the study. The study was undertaken in full accordance with International Conference of Harmonisation Good Clinical Practice guidelines, the Declaration of Helsinki, and other bioethical principles. Eligible patients had histologically or cytologically diagnosed as locally advanced (stage IIIB) or metastatic (stage IV) non-small-cell lung cancer with progression according to the tumour-node-metastasis staging system by the American Joint Committee on Cancer (AJCC). Patients were excluded from the study if they had symptomatic brain metastases, malignant tumour within the previous 5 years, severe infection, congestive heart failure, previous treatment with drugs targeting EGFR, and a history of interstitial lung disease.

Patients accepted $125 \mathrm{mg}$ icotinib T.I.D. treatment and returned to hospital in the $672^{\text {th }}$ and $1680^{\text {th }}$ hour after administration ( $4^{\text {th }}$ week and $10^{\text {th }}$ week). Blood samples were obtained in $672^{\text {th }}$ and $1680^{\text {th }}$ hour, in which plasma used to quantify the concentration of icotinib and blood cells used to check the genotypes. In the meanwhile, patients' clinical data were also collected, including EGFR genotypes.

\section{Plasma samples prepare using SPE extraction column}

SPE column extraction was used to prepare plasma sample. Oasis MCX columns $(30 \mu \mathrm{m}, 10 \mathrm{mg})$ were activated by sequence of $0.5 \mathrm{~mL}$ methanol, $0.5 \mathrm{~mL} 2 \%$ $\mathrm{NH}_{4} \mathrm{OH}$ in methanol and $0.5 \%$ formic acid in water. $140 \mu \mathrm{L}$ plasma samples mixed with $400 \mu \mathrm{L}$ internal standard were added into column. The columns were washed by $0.5 \mathrm{~mL}$ $0.5 \%$ formic acid in water and $10 \%$ water in methanol. At last, elution from $2 \% \mathrm{NH}_{4} \mathrm{OH}$ in water:methanol=1:9

Table 1. Demographic Information of all Patients

\begin{tabular}{lrc}
\hline & Number & Frequency $(\%)$ \\
\hline Gender & & \\
Male & 96 & 61.5 \\
Female & 60 & 38.5 \\
Age & & \\
$\quad<50$ years & 44 & 28 \\
$50-70$ years & 103 & 66 \\
$>70$ years & 9 & 6 \\
Types of carcinoma & & \\
Squamous carcinoma & 27 & 17.3 \\
Adenocarcinoma & 122 & 78.2 \\
Adeno-squamous carcinoma & 6 & 3.8 \\
Large cell carcinoma & 1 & 0.7 \\
Smoking years & & \\
$\quad<10$ years & 8 & 5 \\
10-30 years & 33 & 21 \\
$>30$ years & 47 & 30 \\
No records & 68 & 44 \\
Cigarette amounts per day & & \\
$\quad<10$ cigarettes per day & 8 & 5 \\
10-30 cigarettes per day & 47 & 30 \\
> 30 cigarettes per day & 23 & 15 \\
No records & 78 & 50 \\
\hline
\end{tabular}

was collected, and blew to dry by nitrogen gas at $40^{\circ} \mathrm{C}$. Samples were re-dissolved in $5 \%$ methanol in $10 \mathrm{mM}$ ammonium acetate $(\mathrm{PH}=8)$.

\section{HPLC/MS/MS method to detect plasma concentration of icotinib}

Quantified method using HPLC/MS/MS was developed in Phase I trials as described: The analytes were detected by monitoring icotinib, its main metabolites M20, M23, M24, M26 and internal standard material midazolam, respectively. Plasma samples were analyzed using 2695 Alliance HPLC system (Waters Co., MA, USA) coupled with API 3000 tandem MS (Applied Biosystems, CA, USA). An XTerra RP C18 column $(2.1 \mathrm{~mm} \times 50 \mathrm{~mm}$, $3.5 \mu \mathrm{m})$ protected by XTerra RP C18 guard column $(2.1 \mathrm{~mm} \times 10 \mathrm{~mm}, 3.5 \mu \mathrm{m})$ at ambient temperature were used to separate icotinib from plasma sapmples. The mobile phase was composed of 5 mMammonium acetate (containing $0.1 \%$ formic acid)/acetonitrile $(55 / 45, \mathrm{v} / \mathrm{v})$. The flow rate was $0.2 \mathrm{~mL} / \mathrm{min}$ at the first $1.8 \mathrm{~min}$ and was changed into $0.35 \mathrm{~mL} / \mathrm{min}$ during $1.8-3.5 \mathrm{~min}$. The injection volume was $20 \mu \mathrm{L}$.

\section{gDNA sample preparation and Genotype tests}

gDNA samples were extracted by Blood DNA Extract Kit (Tiangen Biotech Co. Ltd., Beijing, China) . To $200 \mu \mathrm{L}$ of blood cell was added $600 \mu \mathrm{L}$ of cell lysis solution. Sample tubes were mixed and incubated at room temperature (RT) for $10 \mathrm{~min}$. Tubes were spun at $13000 \mathrm{~g}$ for $2 \mathrm{~min}$ and supernatant was decanted and the residual liquid vortexed to resuspend by $200 \mu \mathrm{L}$ proteinase $\mathrm{K}$ solution. Samples were incubated at $65^{\circ} \mathrm{C}$ for 10 min. The supernatant was decanted into fresh tubes and $300 \mu \mathrm{L}$ isopropanol was added for precipitation of nucleic acids. Samples were centrifuged at $12000 \mathrm{~g}$ for $1 \mathrm{~min}$. $70 \%$ ethanol was added to the resulting supernatant and decanted to dryness. The DNA pellet was re-dissovled in $100 \mu \mathrm{L}$ DNA solution overnight at RT. gDNA samples were kept at $4^{\circ} \mathrm{C}$ until analysed.

The primers used to amplify the $2 \mathrm{C} 19 * 2, * 3, * 17$ and 2 C $9 * 3$ were designed by Primer 5.0 software. All primers sequences were listed in Table 2. Primers were synthesized in Shanghai Shenggong Co. Ltd. And the PCR tests were performed in a volume of $50 \mu \mathrm{L}$ containing $1 \mu \mathrm{L}$ template gDNA, $2 \mu \mathrm{L}$ mixture of forward and reverse primers, $25 \mu \mathrm{L} 2 \times \mathrm{Taq}$ enzyme mix with $\mathrm{Mg}^{2+}$ and dNTP (Tiangen Co. Ltd., Beijing, China), and $22 \mu \mathrm{LddH} 2 \mathrm{O}$. The reactions were initiated with a denaturation at $95^{\circ} \mathrm{C}$ for 5 min, followed by 38 cycles of $95^{\circ} \mathrm{C}$ for $30 \mathrm{sec}$, different annealing temperature for $30 \mathrm{sec}$ (Table 1 ), $72^{\circ} \mathrm{C}$ for $45 \mathrm{sec}$, and a final extension at $72^{\circ} \mathrm{C}$ for $7 \mathrm{~min}$. The result of agarose gel electrophoresis and sequencing implied the specificity of primers and fragments.

\section{Statistics and Data Analysis}

Demographic results were concluded by descriptive statistics. Genotype frequencies based on the HardyWeinberg equilibrium were checked by Fisher's exact test with a level of significance of 5\%. Bivariate correlations were assessed using the Pearson correlation coefficient. Kaplan-Meier analyses were used to estimate median PFS 
Table 2. Maximum Likelihood Estimates Method to Analysis the Relationship of PFS, OS, Tumor Shrinkage Rate and Plasma Drug Concentration

\begin{tabular}{lccccc}
\hline Dependent Variable & Parameter & "Parameter Estimation & SD & $p$ value & Hazard ratio(95\%CI) \\
\hline PFS & icotinib & -0.745 & 0.312 & $* 0.0169$ & $0.475(0.258,0.875)$ \\
OS & icotinib & -0.729 & 0.311 & $* 0.0193$ & $0.483(0.262,0.888)$ \\
Tumor Shrinkage rate & icotinib & -0.0444 & 0.353 & 0.9 & $0.957(0.479,1.909)$ \\
& M20 & -0.00552 & 0.382 & 0.989 & $0.994(0.470 .2 .103)$ \\
& M23 & -0.18 & 0.381 & 0.637 & $0.835(0.396,1.763)$ \\
& M24 & -0.00696 & 0.389 & 0.986 & $0.993(0.463,2.129)$ \\
& M26 & -0.263 & 0.444 & 0.553 & $1.301(0.545,3.102)$ \\
\hline
\end{tabular}

"parameter estimates in COX model: $\mathrm{f}(\mathrm{y}, \mathrm{x})=\mathrm{f}_{0}(\mathrm{y}) * \exp \left(\beta_{1} \cdot \mathrm{X}_{1}+\beta_{2} \cdot \mathrm{X}_{2}+\cdots+\beta_{\mathrm{n}} \cdot \mathrm{X}_{\mathrm{n}}\right) ;{ }^{*} p<0.05$ showed significant difference
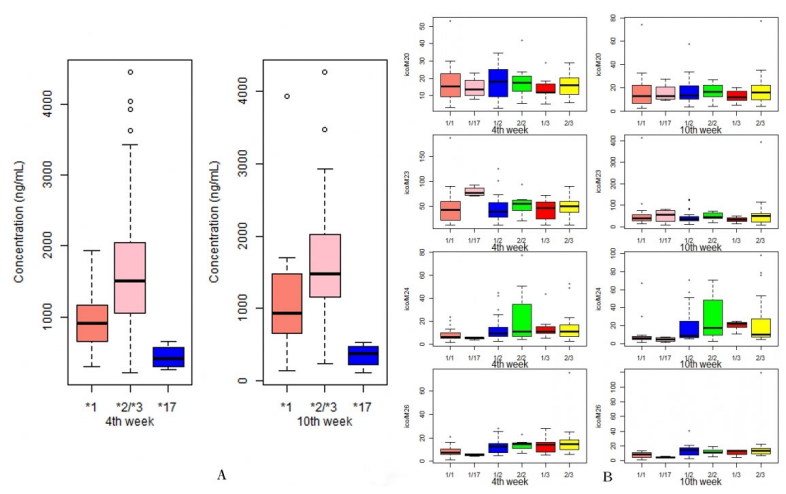

Figure 1.A) Influence of CYP2C19 on Icotinib Plasma Concentration in $4^{\text {th }}$ and $10^{\text {th }}$ week; $B$ Comcentration of Icotinib and its Metabolites M20 M23 M24 M26 in Different CYP2C19 Genotypes Patients Patients Gatients Group in $4^{\text {th }}$ and $10^{\text {th }}$ week

and OS, and the log-rank test and Maximum Likelihood Estimate were used to assess the difference between mutant and non-mutant EGFR groups. A multivariate logistic regression model was applied to determine risk factors for icotinib and estimate the adjusted $95 \%$ confidence interval (CI). A two-tailed $p$-value of less than 0.05 was considered significant. All statistical analyses were performed with SPSS 17.0 and SAS 9.2.

\section{Results}

\section{Demographic and genotype information}

Clinical and demographic data 156 patients aging from 28 to 75 could be collected, which demographic datasets are shown in Table 1. Most patients suffered adenocarcinoma. Although not all the information was recorded, for example EGFR genotypes, smoking years and cigarette amounts per day of some patients, the recorded data exhibited most patients smoking exceeding 10 cigarettes per day in more than 10 years. 312 plasma samples were used to detect concentration of icotinib and its metabolites M20, M23, M24, M26, and 156 gDNA samples were used to check CYP2C19 genotype. The population was divided into 7 genotypes of CYP2C19. 2C19*1, $* 2, * 3$ and $* 17$ shows frequency of $57 \%, 32 \%, 14 \%$ and $1.6 \%$, respectively. Besides CYP 2C19 genotypes, some EGFR genotypes information were also collected that most of Chinese are non-mutant EGFR patients (Table 1).

2C19 genotypes versus icotinib and metabolites exposure Icotinib and metabolites exposure of all patients were successfully detected. Mean concentration with error bar indicating patients with different genotypes shows in Figure 1A. For icotinib concentration, patients carrying poor metabolism genotypes $2 \mathrm{C} 19 * 2$ or $* 3$ exhibited 1.9-fold higher in 672th hour and 1.44-fold higher in 1680th hour than patients with genotype of $* 1 / * 1$, but it became 2.17-fold lower in 672th hour and 3.45-fold lower in 1680th hour compared with wild-type patients. For metabolites exposure, there were not significantly differences on M20 and M23 in all patients with genotypes. On the other hand, compared with patients with genotype of $* 1 / * 1$, higher plasma concentration of M24 and M26 existed in the patients with genotypes of $* 1 / * 2, * 1 / * 3, * 2 / * 2$ and $* 2 / * 3$. On contrary, not significant less M24 and M26 concentration stayed in patients with genotype of $* 1 / * 17$ (shown in Figure 1B).

Icotinib and metabolites exposure versus PFS, OS, tumor shrinkage rate and tumor metastasis

The relationship between exposure of icotinib and metabolites and progressive survival time (PFS) and overall survival (OS) time was assessed by maximum likelihood estimates (Table 2). Patients who had a higher plasma concentration of icotinib live a longer PFS and OS $(p<0.05)$, which can decrease occurrence of death (HR: 0.0169 and 0.0173). Besides, higher icotinib exposure can well inhibit tumor cell metastasis. Accompany with the numbers of tumor metastasis position increasing, the median icotinib concentration also significantly got higher. When concentration was more than 1000ng/ $\mathrm{mL}$, possibility of tumor cell run to liver, brain, bone and epinephros would decrease more than 2-fold, and some of them were completely inhibited, but it seem to be uncorrelated with metabolites exposure (shown in Figure 2). In these patients, tumor size was kept stable or increased in $32.9 \%$ patients and some were shrinkage in $67.1 \%$ patients in which tumor shrinkage rates were from $80 \%$ to $4.3 \%$. However, tumor shrinkages of treated patients were independent of compounds exposure (Table 2).

EGFR genotypes versus PFS, OS and therapeutic status

As previous studies proved that EGFR genotypes of 19del and L858R were significantly related to the therapeutic effect of EGFR-TKI, our results also concluded that EGFR genotype in most partial release (PR) patients was $19 \mathrm{del}$. Only 15 patients showed PR status, including $1 \mathrm{~L} 858 \mathrm{R}$ patient $(7 \%)$ and 14 19del patients (93\%) till $10^{\text {th }}$ week. The rest patients kept on stable disease (SD) 
Table 3. Comparison of Icotinib and its Metabolites Concentration between Adverse Drug Reactions Groups and Relative no Adverse Reaction Groups

\begin{tabular}{|c|c|c|c|c|}
\hline Adverse reaction type & Compounds & $\begin{array}{c}\text { Average concentration in } \\
\text { adverse reaction group }(\mathrm{ng} / \mathrm{mL})\end{array}$ & $\begin{array}{l}\text { Average concentration in } \\
\text { no reaction group }(\mathrm{ng} / \mathrm{mL})\end{array}$ & $p$ value \\
\hline \multirow[t]{10}{*}{ Liver adverse reactions $^{\mathrm{c}}$} & \multirow[t]{2}{*}{ Icotinib } & a4W: 1867.0 & 4W: 1315.8 & 0.04 \\
\hline & & b10W: 1689.2 & 10W: 1332.2 & 0.12 \\
\hline & \multirow[t]{2}{*}{ M20 } & 4W: 146.7 & 4W: 90.3 & $* 0.034$ \\
\hline & & 10W: 193.0 & 10W: 104.3 & $* 0.015$ \\
\hline & \multirow[t]{2}{*}{ M23 } & $4 \mathrm{~W}: 51.4$ & $4 \mathrm{~W}: 32.3$ & $* 0.037$ \\
\hline & & 10W: 60.6 & 10W: 37.2 & $* 0.039$ \\
\hline & \multirow[t]{2}{*}{ M24 } & 4W: 144.1 & 4W: 171.4 & 0.56 \\
\hline & & $10 \mathrm{~W}: 124.0$ & 10W: 153.7 & 0.41 \\
\hline & \multirow[t]{2}{*}{ M26 } & 4W: 183.9 & 4W: 135.7 & 0.23 \\
\hline & & 10W: 156.3 & 10W: 145.6 & 0.76 \\
\hline \multirow[t]{10}{*}{ Gastrointestinal adverse reactions ${ }^{\mathrm{d}}$} & \multirow[t]{2}{*}{ Icotinib } & $4 \mathrm{~W}: 1318.7$ & $4 \mathrm{~W}: 1470.4$ & 0.51 \\
\hline & & 10W: 1336.0 & 10W: 1429.9 & 0.63 \\
\hline & \multirow[t]{2}{*}{ M20 } & 4W: 95.9 & 4W: 103.5 & 0.74 \\
\hline & & 10W: 96.4 & $10 \mathrm{~W}: 132.2$ & 0.25 \\
\hline & \multirow[t]{2}{*}{ M23 } & $4 \mathrm{~W}: 31.5$ & 4W: 41.8 & 0.29 \\
\hline & & $10 \mathrm{~W}: 31.5$ & $10 \mathrm{~W}: 46.4$ & 0.12 \\
\hline & \multirow[t]{2}{*}{ M24 } & 4W: 144.7 & 4W: 175.9 & 0.43 \\
\hline & & 10W: 128.6 & 10W: 159.3 & 0.3 \\
\hline & \multirow[t]{2}{*}{ M26 } & $4 \mathrm{~W}: 156.0$ & 4W: 139.0 & 0.62 \\
\hline & & 10W: 154.2 & 10W: 144.5 & 0.74 \\
\hline \multirow[t]{10}{*}{ Renal adverse reactions $\mathrm{s}^{\mathrm{e}}$} & \multirow[t]{2}{*}{ Icotinib } & 4W: 1094.2 & 4W: 1440.1 & 0.42 \\
\hline & & 10W: 1149.4 & 10W: 1415.5 & 0.46 \\
\hline & \multirow[t]{2}{*}{ M20 } & 4W: 46.6 & 4W: 104.5 & 0.17 \\
\hline & & 10W: 70.9 & 10W: 124.1 & 0.36 \\
\hline & \multirow[t]{2}{*}{ M23 } & 4W: 18.3 & 4W: 40.0 & 0.22 \\
\hline & & $10 \mathrm{~W}: 33.0$ & $10 \mathrm{~W}: 42.1$ & 0.61 \\
\hline & \multirow[t]{2}{*}{ M24 } & 4W: 125.5 & $4 \mathrm{~W}: 169.2$ & 0.55 \\
\hline & & 10W: 162.4 & 10W: 147.2 & 0.79 \\
\hline & \multirow[t]{2}{*}{ M26 } & 4W: 82.6 & 4W: 148.9 & 0.29 \\
\hline & & 10W: 133.7 & 10W: 148.6 & 0.79 \\
\hline
\end{tabular}

${ }^{\mathrm{a}} 4 \mathrm{~W}=4^{\text {th }}$ week; $\mathrm{b} .10 \mathrm{~W}=10^{\text {th }}$ week; ${ }^{\mathrm{c}}$ liver adverse reaction contained AST/ALT elevation liver function injury etc; ${ }^{\mathrm{d}}$ gastrointestinal drug reaction contained diarrhea etc; ${ }^{\mathrm{c}}$ renal drug reaction contained proteinuria, creatinine elevation etc; $* p<0.05$ in both $4^{\text {th }}$ week and $10^{\text {th }}$ week exhibited significant higher exposure in adverse drug reaction group
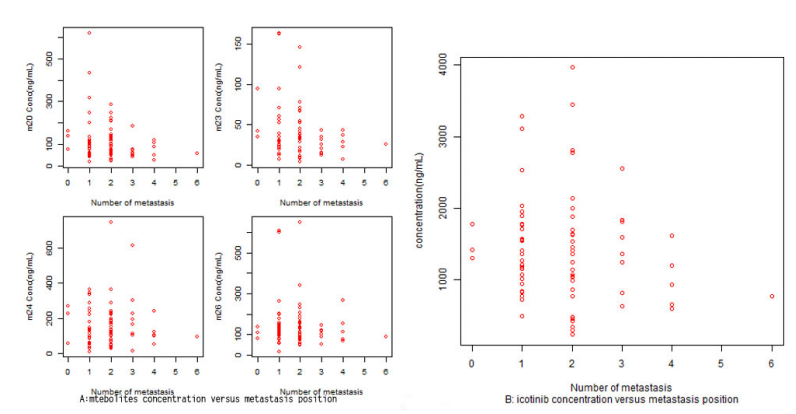

Figure 2. Mean Concentration of Icotinib and its Metabolites Cersus Munbers of Tumor Cell Metastasis Position

or progressive disease (PD). The Kaplan-Meier survival analysis (shown in Figure 3) results illustrated patients with mutant EGFR gene have a significantly higher median PFS 234 days and OS 627 days than who without mutant EGFR having median PFS 108 days and OS 367 days (log rank $p=0.061$ and 0.025 respectively).

Icotinib and metabolites exposure versus toxicity events

As the most adverse drug reaction (ADR) TKI agents had, skin toxicity $(59.21 \%)$ such as rash was main ADR events. Besides, there were some liver (19.74\%), gastrointestinal $(28.95 \%)$ and renal $(9.21 \%)$ adverse events occurred in this phase III trial, for example diarrhea,


Figure 3. Kaplan-Meier Analysis of Progressive-Free and Overall Survival According to EGFR Mutation Status. A). Kaplan-Meier curve for Progressive-Free Survival Days; B). Kaplan-Meier Curve for Overall Survival Days

AST/ALT elevation, proteinuria, creatinine elevation etc. The relationship between icotinib and metabolites exposure and ADR events was shown in Table 3. Patients who suffered liver adverse reaction appeared to have a significant average higher exposure of M20 and M23. Except liver toxicity, there were no significant differences for all exposure in patients suffering gastrointestinal and renal adverse reactions. Different severity of skin toxicity such as rash, skin itches, hand-foot syndrome were the main adverse events of icotinib. Patients who suffered grade II skin toxicity had a higher icotinib exposure $(2046.59 \pm 990.26 \mathrm{ng} / \mathrm{mL})$ than patients who suffered grade I skin toxicity $(1307.96 \pm 673.34 \mathrm{ng} / \mathrm{mL})$ or did not suffered this adverse effect $(1291.78 \pm 600.72 \mathrm{ng} / \mathrm{mL})$. 


\section{Discussion}

In this article, possible influenced parameters, CYP2C19 genotypes and EGFR genotypes, which had been published, were linked to assess how these covariates made effects on therapeutics and adverse drug reaction. The influenced factors described whether the PFS or OS, tumor size shrinkage rate, tumor metastasis, and adverse drug syndrome altered, the exposure of icotinib and its metabolites appeared individually different.

Twenty-nine metabolites of icotinib were found in human plasma, urine, and feces, among which M20, M23, M24 and M26 counted around $80 \%$ of the dosing amount in total (Liu et al., 2011). C Ruan reported that icotinib was mainly metabolized by CYP2C19, CYP3A4, and CYP2E1 by microsomes assays, and CYP2C19 polymorphism obviously altered icotinib exposure in healthy male subjects. The wild-type is normally referred to as CYP2C19*1, which is associated with the full metabolic function of the enzyme. Several polymorphisms of the gene are known to be associated with reduced enzymatic activity, which is principally referred to as CYP2C19*2 and CYP2C19*3. It is widely reported that the drugs metabolized by CYP2C19 have variable plasma concentration, and may be the main factor to influence the therapeutic outcome and cause adverse events. In this study, we further confirmed the influence of CYP2C19 in patients: Compared with patients without mutant gene, lower exposure is presented in subjects carrying ultra-metabolism gene, and the higher concentration is exhibited to subjects carrying poor-metabolism gene. Genetic polymorphism of CYP 2C19 perhaps make an influence on bio-transformation from icotinib to M24 and M26, especially genotypes which lead to poor metabolism.

Although CYP2C19 genetic polymorphism exhibited a big effect on exposure of icotinib, M24 and M26, our study displayed it would not affect the tumor shrinkage. This reason might contribute to the dose of $125 \mathrm{mg} / \mathrm{t}$.i.d which can stay in the widely therapeutic window of icotinib and the therapeutic target EGFR pathway were much dependent with the mutant genotype. Nevertheless, higher icotinib plasma concentration in vivo can well protect can reduce $50 \%$ ineffectiveness or death more or less, even kept the patients live longer. Moreover, inhibition of tumor metastasis was realized when icotinib exposure was more than $1000 \mathrm{ng} / \mathrm{mL}$. As we know, worst situation of cancer is the tumor transferred into bone, brain and other places, so it is probable to prevent tumor transfer while take icotinib as early as possible, especially $2 \mathrm{C} 19 * 2$ or $* 3$ patients. Accompanying with therapeutic effect, adverse drug reaction also turned up based on any known and unknown mechanisms. Our data implied higher icotinib exposure induced severe skin toxicity, such as rash appearing on hands, mouth, face and whole body. In addition, liver toxicity such as AST/ALT elevation occurred in patients in whom there were more M20 and M23 staying in vivo.

In general, EGFR-TKIs maintenance therapy as a second-line therapy to improve ORR and PFS for patients with advanced NSCLC, or as monotherapy was better sometimes (Alimujiang et al., 2013), but a meta-analysis proved that it is unable to prolong patients' OS (Qi et al., 2012). The main adverse reactions were diarrhea and rashes. The reason was explained that EGFR mutations were associated with better survival and a clinical response with EGFR TKIs than were non-EGFR mutations. Patients in the ISEL trial who had an objective response with gefitinib were significant higher in patients with an EGFR mutation $(37.5 \%$ ) than in patients without an EGFR mutation (2.6\%) (Hirsch et al., 2006). A study also showed that patients with EGFR mutations seem to have longer survival than patients with wild-type EGFR when receiving icotinib or gefitinib (Shi et al., 2013, Takano et al., 2008). In our study, we observed patients live a longer PFS or OS with mutant EGFR, especially the genotype of $19 \mathrm{del}$. This finding was supported by a retrospective study assessing the efficacy of icotinib in patients with an EGFR mutation (Song et al., 2013). Except EGFR genotypes, we discovered icotinib exposure had an effect on survival time of patients $(p<0.05)$. But how much icotinib run into tumor cell could make patients' lifespan longer is still unknown and we will plan to build up an icotinib pharmacolinetics-tumor size-survival model in future to quantitatively assess the relationship. In addition, we tried to compare icotinib and metabolites exposure in patients with mutant EGFR. As to tumor shrinkage rates, it was not significant increased in more icotinib exposure than less exposure which was than $1400 \mathrm{ng} / \mathrm{mL}(p>0.05$, data not shown). Furthermore, we also analyzed that exposure under therapeutic dose of icotinib was nothing linked with anti-tumor effect in all EGFR mutations though EGFR genotypes.

Even we tried to discover the relationship between icotinib's and metabolites' exposure-therapeutic-adverse drug effect, but there were several limitations resisting in our study. First, sample size was too small which may make a little influence on PFS assessment. Second, EGFR genotype information was not fully obtained owing to clinical limits. Due to the difficulty of EGFR detect, some researches tried to find some possible factors related to EGFR mutations. EGFR mutation status significantly associated with lung cancer with female patients, pure or mixed ground grass opacity (GGO), adenocarcinoma, never-smoker, smaller tumor diameter in chest CT but never smoking status was the only independent predictor for the presence of EGFR mutations (Alimujiang et al., 2013, Liam et al., 2014, Usuda et al., 2014). Besides, serum CEA and CA242 levels were associated with mutations of the EGFR gene in patients with lung adenocarcinomas (Pan et al., 2013). Third, although study protocol strictly required the blood sampling time, the exact time were not recorded. We consider it has just little effect on drug plasma concentration because the concentration reached the steady plateau. Fourth, all information we got just have the record in baseline, 4th week and 10th week, we did not got real all information till the trial completed and we did not have the final record. In future, we'll try to build up a pop-PK/PD model and excavate more potentials of icotinib, which is benefit for icotinib's development and clinical usage.

In conclusion, the results of our study illustrated that the reported covariate CYP2C19 genotype can obviously affect icotinib exposure, whose mechanism may attribute 


\section{Jia Chen et al}

to transfer to M24 and M26. Higher plasma concentration of icotinib inhibited tumor metastasis and keep patients live a longer PFS and OS. But under the $125 \mathrm{mg} / \mathrm{t}$.i.d dose, tumor shrinkage was more sensitive to EGFR genotype than drug's exposure. So patients with mutant EGFR, especially $19 \mathrm{del}$, would achieve best effect. As to adverse drug effect, greater exposure of icotinib can increase the possibility of occurrence of severe skin toxicity and greater M20 and M23 exposure may induce production of liver injury.

\section{Acknowledgements}

We give our sincere thankfulness to Dr. Tan Fenlai, Dr. Wang Yinxiang, Dr. Liu Yong and Li Xiaofeng from Zhejiang Beta Co.Ltd who gave us support and data to complete this research.

\section{References}

Alimujiang S, Zhang T, Han ZG, et al. (2013) Epidermal growth factor receptor tyrosine kinase inhibitor versus placebo as maintenance therapy for advanced non-small-cell lung cancer: a meta-analysis of randomized controlled trials. Asian Pac J Cancer Prev, 14, 2413-9.

Camidge DR. (2013) Icotinib: kick-starting the Chinese anticancer drug industry. Lancet Oncol, 14, 913-4.

Gao Z, Chen W, Zhang X, et al. (2013) Icotinib, a potent and specific EGFR tyrosine kinase inhibitor, inhibits growth of squamous cell carcinoma cell line A431 through negatively regulating AKT signaling. Biomed Pharmacother, 67, 351-6.

Hirsch FR, Varella-Garcia M, Bunn PA, et al. (2006) Molecular predictors of outcome with gefitinib in a phase III placebocontrolled study in advanced non-small-cell lung cancer. $J$ Clin Oncol, 24, 5034-42.

Liam CK, Leow HR, How SH, et al. (2014) Epidermal growth factor receptor mutations in non- small cell lung cancers in a multiethnic Malaysian patient population. Asian Pac J Cancer Prev, 15, 321-6.

Liu D, Jiang J, Zhang L, et al. (2011) Metabolite characterization of a novel anti-cancer agent, icotinib, in humans through liquid chromatography/quadrupole time-of-flight tandem mass spectrometry. Rapid Commun Mass Spectrom, 25, 2131-40.

Liu D, Jiang J, Zhang L, et al. (2014) Clinical pharmacokinetics of Icotinib, an anti-cancer drug: evaluation of dose proportionality, food effect, and tolerability in healthy subjects. Cancer Chemother Pharmacol, (in press).

Mu X, Zhang Y, Qu X, et al. (2013) Ubiquitin ligase Cbl-b is involved in icotinib (BPI-2009H)-induced apoptosis and G1 phase arrest of EGFR mutation-positive non-small-cell lung cancer. Biomed Res Int, 2013, 726375.

Pan JB, Hou YH, Zhang GJ. (2013) Correlation between EGFR mutations and serum tumor markers in lung adenocarcinoma patients. Asian Pac J Cancer Prev, 14, 695-700.

Qi WX, Shen Z, Lin F, et al. (2012) Comparison of the efficacy and safety of EFGR tyrosine kinase inhibitor monotherapy with standard second-line chemotherapy in previously treated advanced non-small-cell lung cancer: a systematic review and meta-analysis. Asian Pac J Cancer Prev, 13, 5177-82.

Ruan CJ, Liu DY, Jiang J, et al. (2012) Effect of the CYP2C19 genotype on the pharmacokinetics of icotinib in healthy male volunteers. Eur J Clin Pharmacol, 68, 1677-80.

Shao L, Zhang B, He C, et al. (2014) Efficacy and safety of icotinib in Chinese patients with advanced non-small cell lung cancer after failure of chemotherapy. Chin Med J, 127, 266-71.

Shi Y, Zhang L, Liu X, et al. (2013) Icotinib versus gefitinib in previously treated advanced non-small-cell lung cancer (ICOGEN): a randomised, double-blind phase 3 noninferiority trial. Lancet Oncol, 14, 953-61.

Song Z, Yu X, Cai J, et al. (2013) [Efficacy of icotinib for advanced non-small cell lung cancer patients with EGFR status identified]. Zhongguo Fei Ai Za Zhi, 16, 138-43.

Takano T, Fukui T, Ohe Y, et al. (2008) EGFR mutations predict survival benefit from gefitinib in patients with advanced lung adenocarcinoma: a historical comparison of patients treated before and after gefitinib approval in Japan. J Clin Oncol, 26, 5589-95.

Tan F, Shen X, Wang D, et al. (2012) Icotinib (BPI-2009H), a novel EGFR tyrosine kinase inhibitor, displays potent efficacy in preclinical studies. Lung Cancer, 76, 177-82.

Usuda K, Sagawa M, Motono N, et al. (2014) Relationships between EGFR mutation status of lung cancer and preoperative factors - are they predictive? Asian Pac J Cancer Prev, 15, 657-62.

Wang HP, Zhang L, Wang YX, et al. (2011) Phase I trial of icotinib, a novel epidermal growth factor receptor tyrosine kinase inhibitor, in Chinese patients with non-small cell lung cancer. Chin Med J, 124, 1933.

Zhao Q, Shentu J, Xu N, et al. (2011) Phase I study of icotinib hydrochloride (BPI-2009H), an oral EGFR tyrosine kinase inhibitor, in patients with advanced NSCLC and other solid tumors. Lung Cancer, 73, 195-202. 